\title{
Distribution and genetic characterization of Mycobacterium chelonae in laboratory zebrafish Danio rerio
}

\author{
Christopher M. Whipps ${ }^{1, *}$, Jennifer L. Matthews ${ }^{2}$, Michael L. Kent ${ }^{3}$ \\ ${ }^{1}$ State University of New York College of Environmental Science and Forestry, Environmental and Forest Biology, \\ 246 Illick Hall, 1 Forestry Drive, Syracuse, New York 13210, USA \\ ${ }^{2}$ Zebrafish International Resource Center, 5274 University of Oregon, Eugene, Oregon 97403-5274, USA \\ ${ }^{3}$ Center for Fish Disease Research, Department of Microbiology, 220 Nash Hall, Oregon State University, Corvallis, \\ Oregon 97331-3404, USA
}

\begin{abstract}
During routine screening of zebrafish at a research facility, histological changes consistent with mycobacteriosis were observed, prompting an investigation to determine the background prevalence and distribution of Mycobacterium species throughout the facility. Infection status was evaluated in 240 zebrafish representing 9 genetic lines, using histology, culture and PCR. Environmental sources were also tested for the presence of mycobacteria. Prevalence in zebrafish by culture and PCR was $10 \%$ (24/240), 21 of which were TU line fish. All isolates from fish were identified as $M$. chelonae by hsp65 DNA sequencing; subsequent DNA fingerprinting delineated 3 strains, designated H1E1 (1/24), H1E2 (22/24), and H1E3 (1/24). From external sources, tank or tubing surface biofilms were positive by culture (13/32) with multiple species and strains isolated including $M$. neoaurum, $M$. phocaicum, and identical strains of $M$. chelonae that were found in zebrafish: H1E1 (2/13) and H1E2 (8/13). Comparing diagnostic methods, acid-fast stained histological sections showed substantial agreement with plate culture and PCR for detection of mycobacteria in fish. Observation of granulomas in hematoxylin and eosin-stained sections was a less reliable predictor of mycobacteriosis, as uninfected females with egg-associated inflammation and hyperplasia were misdiagnosed. These data revealed background levels of mycobacteriosis in a healthy and wellmanaged facility. Infected populations were removed, although the apparent ability for $M$. chelonae to remain viable in environmental reservoirs may make it difficult to eradicate completely. This highlights the importance of an animal-health monitoring program and good husbandry practices to prevent disease in zebrafish research laboratories.
\end{abstract}

KEY WORDS: Mycobacterium chelonae - Zebrafish • Danio rerio - Biofilms · Enterobacterial repetitive intergenic consensus PCR · ERIC-PCR · Randomly amplified polymorphic DNA · RAPD

Resale or republication not permitted without written consent of the publisher

\section{INTRODUCTION}

Mycobacteriosis is recognized as a significant disease problem in zebrafish research facilities (Astrofsky et al. 2000, Kent et al. 2004, Whipps et al. 2007a). Findings range from overt disease outbreaks with high mortality to incidental findings where few fish show signs of disease. Several species of Mycobacterium have been identified, and experimental exposures indicate dramatic differences between species and strains (Watral \& Kent 2007). Some M. marinum strains cause more disease than others (Ostland et al. 2008), and even modest exposures to $M$. haemophilum causes overt disease and mortality (Whipps et al. 2007a). We have also isolated $M$. chelonae, $M$. abscessus, and $M$. peregrinum from zebrafish during disease outbreaks (Kent et al. 2004), but little disease or mortality occurred when fish were exposed experimentally (Watral \& Kent 2007). Thus, although some species of mycobacteria cause outright disease, other species 
infect with low virulence and the opportunistic manifestation of disease may be a result of suboptimal environmental conditions.

Even in the absence of mortalities, chronic underlying infections are a threat to the efficacy of results derived from experiments that employ zebrafish. As with any research animal (Baker 2003), it is imperative that disease be minimized. Acute diseases causing severe morbidity and mortality in zebrafish have been reported (Kent et al. 2004, Whipps et al. 2007a), but little is known about the distribution and prevalence of sub-clinical infections. Good husbandry practices likely minimize the potential spread of pathogens in a system, and when standard practices include a health monitoring program, infected stocks can be identified early and isolated or removed. Case in point, in January 2006, during routine monitoring at a zebrafish research laboratory, a few fish exhibiting lesions consistent with mycobacteriosis were identified and the infection was confirmed by histology. This prompted the removal of the stock and further evaluation of the presence of background levels of Mycobacterium spp. infections throughout the facility.

\section{MATERIALS AND METHODS}

Facility. All fish that were introduced into the facility were derived from eggs disinfected with chlorine as described by Westerfield (2000). The facility has a traditional configuration with multiple tanks receiving recirculated water from a filtration system in which nitrogenous wastes were removed with large sandbased biological filters; then water was disinfected by ultraviolet sterilization. Water was held at 28 to $28.5^{\circ} \mathrm{C}$, pH 6.8 to 7.2 , conductivity $500 \mu \mathrm{S}$. Fish were fed twice daily, once with brine shrimp nauplii and once with a commercial flake diet mix.

Survey. Between January and April 2006, 240 zebrafish representing 9 genetic lines (TU, TL, mitfa, sparse, cct2, brg1, Tg(hsp701:Gal4)1.5kca4, WIK, and AB) were tested for mycobacterial infections by histology, culture and PCR (Table 1). Individuals were euthanized with an overdose of tricane methanesulfonate (Argent Laboratories) and examined for clinical signs of mycobacteriosis, i.e. presence of dermal lesions, emaciation and raised scales. The external surface was then cleaned with ethanol and a laboratory tissue in preparation for necropsy. For each fish, the peritoneal cavity was opened along the lateral line with sterile instruments, being careful to avoid contact with visceral organs. At this point, the presence of any visible granulomas was noted. Using another set of sterile instruments, a portion of the spleen and liver were removed and placed in a tube containing $100 \mu \mathrm{l} 1 \%$ cetyl pyridinium chloride (CPC) for culture. An additional piece of spleen or liver was removed and frozen at $-20^{\circ} \mathrm{C}$ for PCR. The remainder of the zebrafish was placed in Dietrich's fixative for histological processing.

Environmental sources. Food supply, tank surfaces, tubing, water reservoirs, and sources outside of the building were examined for the presence of mycobacteria (Table 2). Water was collected in $2 \mathrm{ml}$ tubes, contents pelleted at $10000 \times g$ for $10 \mathrm{~min}$, supernatant carefully removed, and pellets treated overnight in $100 \mu \mathrm{l} 1 \%$ CPC prior to culture. Surfaces were tested using a sterile swab, which was placed directly in $200 \mu \mathrm{l}$ of $1 \%$ CPC overnight and processed for culture.

Culture and histology. All samples treated with $1 \%$ CPC overnight were processed for culture following Kent \& Kubica (1985). Cultures were grown on Middlebrook (MB) 7H10 biplates and Lowenstein-Jensen growth medium (LJ), incubated at $29^{\circ} \mathrm{C}$, and monitored for growth for up to $8 \mathrm{wk}$. Rapid fungal overgrowth in water samples collected from outside of the facility required a second attempt at culture on $\mathrm{MB}$ $7 \mathrm{H} 10$, supplemented with $0.25 \mu \mathrm{g} \mathrm{ml}^{-1}$ amphotericin B.

For histology, fish were preserved whole in Dietrich's fixative. For decalcification prior to histological processing, the tissues were transferred to trifluoroacetic acid (5\%) for $24 \mathrm{~h}$. Fish were then processed using standard methods and stained with hematoxylin and eosin (H\&E), Ziehl-Neelsen's acid-fast, or Kinyoun's acid-fast. Multiple sagittal sections were prepared from each fish, allowing visual evaluation of essentially all visceral organs. Agreement between methods was evaluated using Cohen's unweighted Kappa (Cohen 1960), and categorized by the standards proposed by Landis \& Koch (1977) as follows: Kappa coefficient $K: \leq 0=$ poor, 0.01 to $0.2=$ slight, 0.21 to $0.4=$ fair, 0.41 to $0.6=$ moderate, 0.61 to $0.8=$ substantial, and 0.81 to $1=$ almost perfect.

Molecular. Genomic DNA from biofilms and spleen or liver tissue was extracted using the DNeasy Tissue Kit (QIAGEN) following pretreatment with $20 \mathrm{mg} \mathrm{ml}^{-1}$ lysozyme (Sigma) for $1 \mathrm{~h}$ at $37^{\circ} \mathrm{C}$. For cultures, DNA was extracted from 3 individual colonies grown in $7 \mathrm{H} 9$ broth, using the UltraClean microbial DNA isolation kit (MoBio Laboratories). For extracted tissues and cultures, PCR was used to amplify heat shock protein 65 (hsp65) genes using conditions and primers as described previously (Kent et al. 2004, Poort et al. 2006, Whipps et al. 2007b). Following gel electrophoresis, PCR products were gel-extracted using the QIAquick Gel Extraction Kit (QIAGEN). Direct sequencing was carried out using amplification primers with the ABI BigDye Terminator Cycle Sequencing Ready Reaction Kit v3.1, using the ABI PRISM ${ }^{\circledR} 3730$ DNA Analyzer (Applied Biosystems). Enterobacterial repetitive intergenic consensus (ERIC) PCR was performed following the methods of Sampaio et al. (2006). Additional ran- 
Table 1. Danio rerio examined for Mycobacterium spp. infection with percent positive for histology, culture and PCR combined (Positive by any test). Number of male (M) and female (F) fish for each sample is indicated. Results from histological sections stained with hematoxylin and eosin are indicated: GRAN, granulomas; EAIF, egg-associated inflammation and fibroplasia; AERO, aerocystitis. Results from acid-fast stained sections are: AFB, acid-fast bacilli observed; SB AFB, acid-fast bacilli on the swim bladder wall. Mycobacteria (Myco ID) were identified by hsp65 gene sequencing, and strains of M. chelonae (Mc strain) were further characterized by enterobacterial repetitive intergenic consensus (ERIC) and randomly amplified polymorphic DNA (RAPD) PCR. na: not applicable

\begin{tabular}{|c|c|c|c|c|c|c|c|c|c|c|c|c|c|}
\hline $\begin{array}{l}\text { Date } \\
(\mathrm{d} / \mathrm{mo} / \mathrm{yr})\end{array}$ & Line & $\begin{array}{l}\text { Positive by } \\
\text { any test (\%) }\end{array}$ & $\begin{array}{l}\text { Fish } \\
\text { no. }\end{array}$ & $\mathrm{M}, \mathrm{F}$ & GRAN & EAIF & AERO & AFB & $\begin{array}{l}\mathrm{SB} \\
\mathrm{AFB}\end{array}$ & Culture & $\begin{array}{l}\text { Tissue } \\
\text { PCR }\end{array}$ & $\begin{array}{l}\text { Myco } \\
\text { ID }\end{array}$ & $\begin{array}{c}\text { Mc } \\
\text { strain }\end{array}$ \\
\hline \multirow[t]{6}{*}{ 13/1/06 } & TU & $25(5 / 20)$ & & 13,7 & & & & & & $2 / 20$ & & & \\
\hline & & & ZF-23 & M & + & na & + & + & + & + & + & chelonae & H1E2 \\
\hline & & & $\mathrm{ZF}-24$ & $\mathrm{M}$ & + & na & + & + & + & $\mathrm{Neg}$ & $\mathrm{Neg}$ & na & na \\
\hline & & & ZF-26 & $\mathrm{F}$ & Neg & Neg & Neg & Neg & Neg & + & + & chelonae & H1E2 \\
\hline & & & ZF-37 & $\mathrm{F}$ & + & + & $\mathrm{Neg}$ & $\mathrm{Neg}$ & $\mathrm{Neg}$ & Neg & Neg & na & na \\
\hline & & & ZF-39 & $\mathrm{M}$ & + & na & + & + & + & $\mathrm{Neg}$ & $\mathrm{Neg}$ & na & na \\
\hline \multirow[t]{8}{*}{$31 / 1 / 06$} & $\mathrm{TU}$ & $35(7 / 20)$ & & 16,4 & & & & & & $5 / 20$ & & & \\
\hline & & & ZF-63 & $\mathrm{M}$ & + & na & + & + & + & + & + & chelonae & H1E2 \\
\hline & & & ZF-70 & $\mathrm{F}$ & + & + & Neg & $\mathrm{Neg}$ & $\mathrm{Neg}$ & Neg & Neg & na & na \\
\hline & & & ZF-71 & $\mathrm{F}$ & + & + & Neg & Neg & $\mathrm{Neg}$ & $\mathrm{Neg}$ & Neg & na & na \\
\hline & & & ZF-72 & $\mathrm{M}$ & + & na & + & + & + & + & + & chelonae & H1E2 \\
\hline & & & ZF-75 & $\mathrm{M}$ & + & na & + & + & + & + & + & chelonae & H1E2 \\
\hline & & & ZF-79 & $\mathrm{M}$ & + & na & + & + & + & + & + & chelonae & H1E2 \\
\hline & & & ZF-80 & $\mathrm{M}$ & + & na & + & + & + & + & + & chelonae & H1E2 \\
\hline \multirow[t]{5}{*}{$31 / 1 / 06$} & TU & $20(4 / 20)$ & & 16,4 & & & & & & $3 / 20$ & & & \\
\hline & & & ZF-45 & F & + & + & Neg & Neg & $\mathrm{Neg}$ & Neg & Neg & na & na \\
\hline & & & $\mathrm{ZF}-48$ & $\mathrm{M}$ & Neg & na & Neg & $\mathrm{Neg}$ & Neg & + & + & chelonae & H1E2 \\
\hline & & & ZF-55 & $\mathrm{F}$ & $\mathrm{Neg}$ & $\mathrm{Neg}$ & $\mathrm{Neg}$ & $\mathrm{Neg}$ & $\mathrm{Neg}$ & + & + & chelonae & H1E1 \\
\hline & & & ZF-60 & $\mathrm{F}$ & + & + & Neg & + & $\mathrm{Neg}$ & + & + & chelonae & H1E2 \\
\hline \multirow[t]{2}{*}{ 07/3/06 } & $\mathrm{TL}$ & $5(1 / 20)$ & & 7,13 & & & & & & $1 / 20$ & & & \\
\hline & & & ZF-84 & $\mathrm{M}$ & Neg & na & Neg & Neg & Neg & + & + & chelonae & H1E2 \\
\hline $13 / 3 / 06$ & mitfa & $0(0 / 20)$ & & 14,6 & & & & & & $0 / 20$ & & & \\
\hline \multirow[t]{3}{*}{$13 / 3 / 06$} & kita & $10(2 / 20)$ & & 7,13 & & & & & & $1 / 20$ & & & \\
\hline & & & ZF-126 & $\mathrm{F}$ & + & + & $\mathrm{Neg}$ & + & $\mathrm{Neg}$ & Neg & $\mathrm{Neg}$ & na & na \\
\hline & & & ZF-128 & $\mathrm{F}$ & + & + & Neg & + & $\mathrm{Neg}$ & + & + & chelonae & H1E2 \\
\hline $21 / 3 / 06$ & cct2 & $0(0 / 20)$ & & 15,5 & & & & & & $0 / 20$ & & & \\
\hline \multirow[t]{12}{*}{$21 / 3 / 06$} & $\mathrm{TU}$ & $55(11 / 20)$ & & 20,0 & & & & & & $11 / 20$ & & & \\
\hline & & & ZF-163 & $\mathrm{M}$ & + & na & + & + & + & + & + & chelonae & H1E2 \\
\hline & & & ZF-164 & $\mathrm{M}$ & + & na & $\mathrm{Neg}$ & + & $\mathrm{Neg}$ & + & + & chelonae & H1E2 \\
\hline & & & ZF-167 & $\mathrm{M}$ & + & na & + & + & + & + & + & chelonae & H1E2 \\
\hline & & & ZF-170 & M & + & na & + & + & + & + & + & chelonae & H1E2 \\
\hline & & & $\mathrm{ZF}-173$ & $\mathrm{M}$ & + & na & + & + & + & + & + & chelonae & H1E2 \\
\hline & & & ZF-175 & $\mathrm{M}$ & + & na & + & + & + & + & + & chelonae & H1E2 \\
\hline & & & ZF-176 & M & $\mathrm{Neg}$ & na & $\mathrm{Neg}$ & Neg & Neg & + & + & chelonae & H1E3 \\
\hline & & & ZF-177 & M & + & na & + & + & + & + & + & chelonae & H1E2 \\
\hline & & & ZF-178 & $\mathrm{M}$ & + & na & + & + & + & + & + & chelonae & H1E2 \\
\hline & & & ZF-179 & M & + & na & + & + & + & + & + & chelonae & H1E2 \\
\hline & & & ZF-180 & $\mathrm{M}$ & + & na & $\mathrm{Neg}$ & + & + & + & + & chelonae & H1E2 \\
\hline \multirow[t]{3}{*}{$04 / 4 / 06$} & brg1 & $10(2 / 20)$ & & 4,16 & & & & & & $0 / 20$ & & & \\
\hline & & & ZF-195 & $\mathrm{F}$ & + & + & Neg & Neg & Neg & Neg & Neg & na & na \\
\hline & & & ZF-200 & $\mathrm{F}$ & + & + & $\mathrm{Neg}$ & $\mathrm{Neg}$ & $\mathrm{Neg}$ & $\mathrm{Neg}$ & $\mathrm{Neg}$ & na & na \\
\hline 04/4/06 & $\begin{array}{c}\text { Tg(hsp 70l:Gal4) } \\
1.5 \mathrm{kca} 4\end{array}$ & $0(0 / 20)$ & & 4,16 & & & & & & $0 / 20$ & & & \\
\hline \multirow[t]{3}{*}{$11 / 4 / 06$} & WIK & $10(2 / 20)$ & & 12,8 & & & & & & $1 / 20$ & & & \\
\hline & & & ZF-225 & $\mathrm{M}$ & $\mathrm{Neg}$ & na & $\mathrm{Neg}$ & $\mathrm{Neg}$ & Neg & + & + & chelonae & H1E2 \\
\hline & & & ZF-231 & F & + & + & $\mathrm{Neg}$ & $\mathrm{Neg}$ & $\mathrm{Neg}$ & Neg & $\mathrm{Neg}$ & na & na \\
\hline \multirow[t]{2}{*}{$20 / 4 / 06$} & $\mathrm{AB}$ & $5(1 / 20)$ & & 10,10 & & & & & & $0 / 20$ & & & \\
\hline & & & ZF-250 & F & + & + & Neg & Neg & $\mathrm{Neg}$ & Neg & Neg & na & na \\
\hline \multicolumn{2}{|c|}{ Total positive } & $35 / 240$ & & 135,105 & 29 & 11 & 16 & 21 & 17 & $24 / 240$ & $24 / 240$ & & \\
\hline
\end{tabular}


Table 2. Locations of environmental and biofilm collections, cultured on MB 7H10. Multiple colony types were initially screened by acid-fast staining to select mycobacteria for subculture. Species were identified by hsp65 gene sequencing, and strains of Mycobacterium chelonae (Mc strain) were further characterized by ERIC and RAPD PCR. AFB: acid-fast bacilli; na: not applicable (1 gal = ca. $3.8 \mathrm{l})$

\begin{tabular}{|c|c|c|c|c|c|c|}
\hline Sample & Location & Colony types & Colony no. & hsp65 sequence & $\begin{array}{c}\text { GenBank } \\
\text { accession no. }\end{array}$ & Mc strain \\
\hline 1 & Paramecium culture & None & $\mathrm{BF}-1$ & na & na & na \\
\hline 2 & Brine shrimp cone & None & $\mathrm{BF}-2$ & na & na & na \\
\hline 3 & Tank, no fish (20 gal) & None & $\mathrm{BF}-3$ & na & na & na \\
\hline \multirow[t]{3}{*}{4} & \multirow[t]{3}{*}{ Side of tank (20 gal) } & \multirow[t]{3}{*}{ Multiple } & $\mathrm{BF}-4 \mathrm{~A}$ & Not AFB & na & na \\
\hline & & & $\mathrm{BF}-4 \mathrm{~B}$ & neoaurum & EU478698 & na \\
\hline & & & $\mathrm{BF}-4 \mathrm{C}$ & neoaurum & EU478697 & na \\
\hline \multirow[t]{2}{*}{5} & \multirow[t]{2}{*}{ Algae/detritis on tank bottom (20 gal) } & \multirow[t]{2}{*}{ Multiple } & BF-5A & chelonae & DQ866784 & H1E2 \\
\hline & & & BF-5B & phocaicum/peregrinum & EU478699 & na \\
\hline \multirow[t]{2}{*}{6} & \multirow[t]{2}{*}{ Side of tank (1 gal) } & \multirow[t]{2}{*}{ Multiple } & $\mathrm{BF}-6 \mathrm{~A}$ & chelonae & DQ866785 & H2E2 \\
\hline & & & $\mathrm{BF}-6 \mathrm{~B}$ & phocaicum/peregrinum & EU478699 & na \\
\hline 7 & Uncleaned tank meniscus (20 gal) & None & BF-7 & na & na & na \\
\hline 8 & Debris on tank bottom (20 gal) & None & $\mathrm{BF}-8$ & na & na & na \\
\hline 9 & Side of tank (20 gal) & Single & BF-9 & chelonae & DQ866784 & H1E2 \\
\hline 10 & Drain bulkhead (20 gal) & Single & $\mathrm{BF}-10$ & chelonae & DQ866784 & H1E1 \\
\hline \multirow[t]{2}{*}{11} & \multirow[t]{2}{*}{ Side of tank (20 gal) } & \multirow[t]{2}{*}{ Multiple } & $\mathrm{BF}-11 \mathrm{~A}$ & chelonae & DQ866784 & H1E2 \\
\hline & & & $\mathrm{BF}-11 \mathrm{~B}$ & Not AFB & na & na \\
\hline 12 & Fungal mat on tank bottom (20 gal) & Single & $\mathrm{BF}-12$ & chelonae & DQ866784 & H1E2 \\
\hline 13 & Clean tank, no fish (1 gal) & None & $\mathrm{BF}-13$ & na & na & na \\
\hline 14 & Side of tank (1 gal) & Single & BF-14 & chelonae & DQ866784 & H1E2 \\
\hline 15 & Side of sump & Single & BF-15 & chelonae & DQ866784 & H1E2 \\
\hline 16 & Side of sump & Single & BF-16 & chelonae & DQ866784 & H1E2 \\
\hline 17 & Clean sump & None & BF-17 & na & na & na \\
\hline 18 & Side of sump & Single & BF-18 & chelonae & DQ866784 & H1E2 \\
\hline 19 & Side of tank (4 gal) & Single & BF-19 & phocaicum & EF551431 & na \\
\hline 20 & Side of tank (1 gal) & None & BF-20 & na & na & na \\
\hline 21 & Main water supply ball valve 1 & None & $\mathrm{BF}-31$ & na & na & na \\
\hline 22 & Main water supply ball valve 2 & None & $\mathrm{BF}-32$ & na & na & na \\
\hline 23 & Norprene tubing from tank & Single & $\mathrm{BF}-33$ & chelonae & DQ866784 & H1E1 \\
\hline 24 & Air filter & None & $\mathrm{BF}-34$ & na & na & na \\
\hline 25 & Air pump & None & $\mathrm{BF}-35$ & na & na & na \\
\hline 26 & Mud puddle & None $^{a}$ & $\mathrm{BF}-36$ & na & na & na \\
\hline 27 & River bank & Single & $\mathrm{BF}-37$ & Not AFB & na & na \\
\hline 28 & Creek 1 & None $^{a}$ & $\mathrm{BF}-38$ & na & na & na \\
\hline 29 & Creek 2 & None $^{a}$ & BF-39 & na & na & na \\
\hline 30 & Lunchroom tap water & None & $\mathrm{BF}-40$ & na & na & na \\
\hline 31 & Sodium thiosulfate tank & Single & $\mathrm{BF}-41$ & Not AFB & na & na \\
\hline 32 & Inside dishwasher & None & BF-42 & na & na & na \\
\hline
\end{tabular}

domly amplified polymorphic DNA (RAPD) primers OPA2, OPA18, IS986-FP and INS-2 (Zhang et al. 1997) were also tested. Amplification patterns were compared visually and relatedness categorized as in Zhang et al. (1997).

\section{RESULTS}

\section{Survey}

While overall mortalities routinely observed in the facility were very low, occurrence of mycobacteriosis in a few fish in a population of some 200 TU zebrafish prompted further investigation. Forty zebrafish from this tank were selected at random for disease screening, 7 of which $(17.5 \%)$ were positive for mycobacteria by culture and PCR testing. Fish from the same stock in an adjacent tank, but not exhibiting signs of disease were also tested and 3/20 (15\%) were positive. When an additional group of $20 \mathrm{TU}$ fish originating from a separate stock held elsewhere in the facility were tested, 11 (55\%) were infected with mycobacteria. Of the remaining groups of zebrafish tested (TL, mitfa, kita, cct2, brg1, Tg(hsp701:Gal4)1.5 kca4, WIK, and $A B)$, only $3 / 160(1.9 \%)$ of these zebrafish were positive 
by culture (Table 1 ). In total, 240 zebrafish were examined with $24(10 \%)$ positive for infection based on culture and PCR of the organism.

\section{Environmental reservoirs}

We were able to culture mycobacteria from a variety of sources around the facility (Table 2). Tank surfaces at the interface of air and water yielded positive results most often, with a variety of Mycobacterium spp. identified. Food preparations (brine shrimp, paramecia) were negative, as were cultures from the main water supply. Multiple species of Mycobacterium were identified in biofilms (see Table 2).

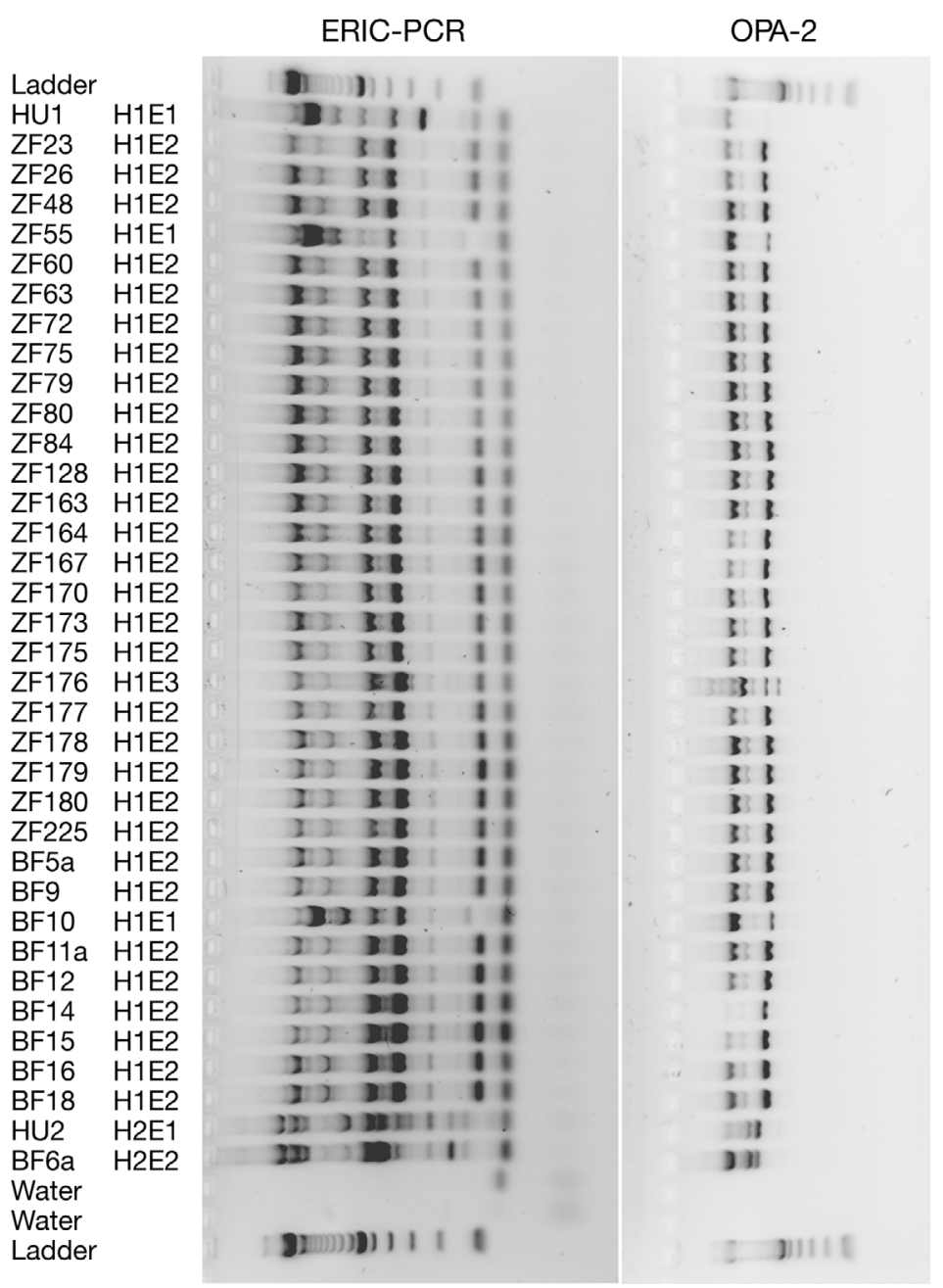

Fig. 1. Mycobacterium chelonae infecting Danio rerio. Amplification products from enterobacterial repetitive intergenic consensus PCR (ERIC-PCR) and randomly amplified polymorphic DNA (RAPD) with primer OPA-2 of $36 \mathrm{M}$. chelonae isolates from zebrafish. HU1 and HU2 were described previously (Kent et al. 2004). Samples designated ZF were colonies isolated from zebrafish, BF were from biofilms. $100 \mathrm{bp}$ DNA ladder in first and last lanes

\section{Molecular characterization}

In all cases where mycobacteria were isolated from zebrafish, hsp65 DNA sequencing identified the isolates as Mycobacterium chelonae (Table 1). Furthermore, all were identical to the hsp65 sequence of $M$. chelonae isolate HU1 (GenBank \# DQ866784) obtained from a zebrafish in our previous work (Kent et al. 2004, Whipps et al. 2007b). All isolates were analyzed further using ERIC-PCR and RAPD primer OPA2 and could be categorized into 3 strains, designated H1E1 (1 isolate), H1E2 (22 isolates), and H1E3 (1 isolate) (Fig. 1). RAPD primers OPA-18, IS986-FP, and INS-2 yielded equivalent or less resolution (data not shown).

From biofilms, multiple species of Mycobacterium (M. chelonae, M. phocaicum, M. neoaurum and an unclassified species) were identified by hsp65 sequencing (Table 2). The hsp65 sequences from these $M$. chelonae isolates was identical to those previously isolated from zebrafish, strains HU1 (GenBank \# DQ866784) and HU2 (GenBank \# DQ866785). Subtyping analysis (Fig. 1) identified the same strains of $M$. chelonae in biofilms, H1E1 (2 isolates) and H1E2 (8 isolates), that were isolated from zebrafish. $\mathrm{My}$ cobacterium species other than $M$. chelonae were not analyzed by ERIC-PCR or RAPD.

\section{Histopathology}

Twenty-nine of 240 zebrafish surveyed exhibited chronic inflammatory changes consistent with mycobacteriosis (Table 1). Most were males ( $n=18$ ) exhibiting moderate to severe, chronic, diffuse inflammation and 16/18 displayed necrosis of the swim bladder (aerocystitis). No aerocystitis was observed in females. The affected swim bladders showed severe necrosis of the epithelium, with inflammatory changes extending through all layers of the swim bladder wall, and accompanying diffuse peritonitis. Acid-fast stains revealed numerous, free acidfast bacilii (AFB) in the lumen of the swim bladder and often numerous bacteria in inflammatory cells in the swim bladder wall and surrounding peritoneum (Fig. 2).

The 11 females presenting with chronic inflammatory changes all had lesions in the ovaries and were associated with degenerated eggs (referred to egg-associated inflammation and fibroplasia, EAIF). In only 3 of these 11 zebrafish did acid-fast staining of sections reveal mycobacteria. 


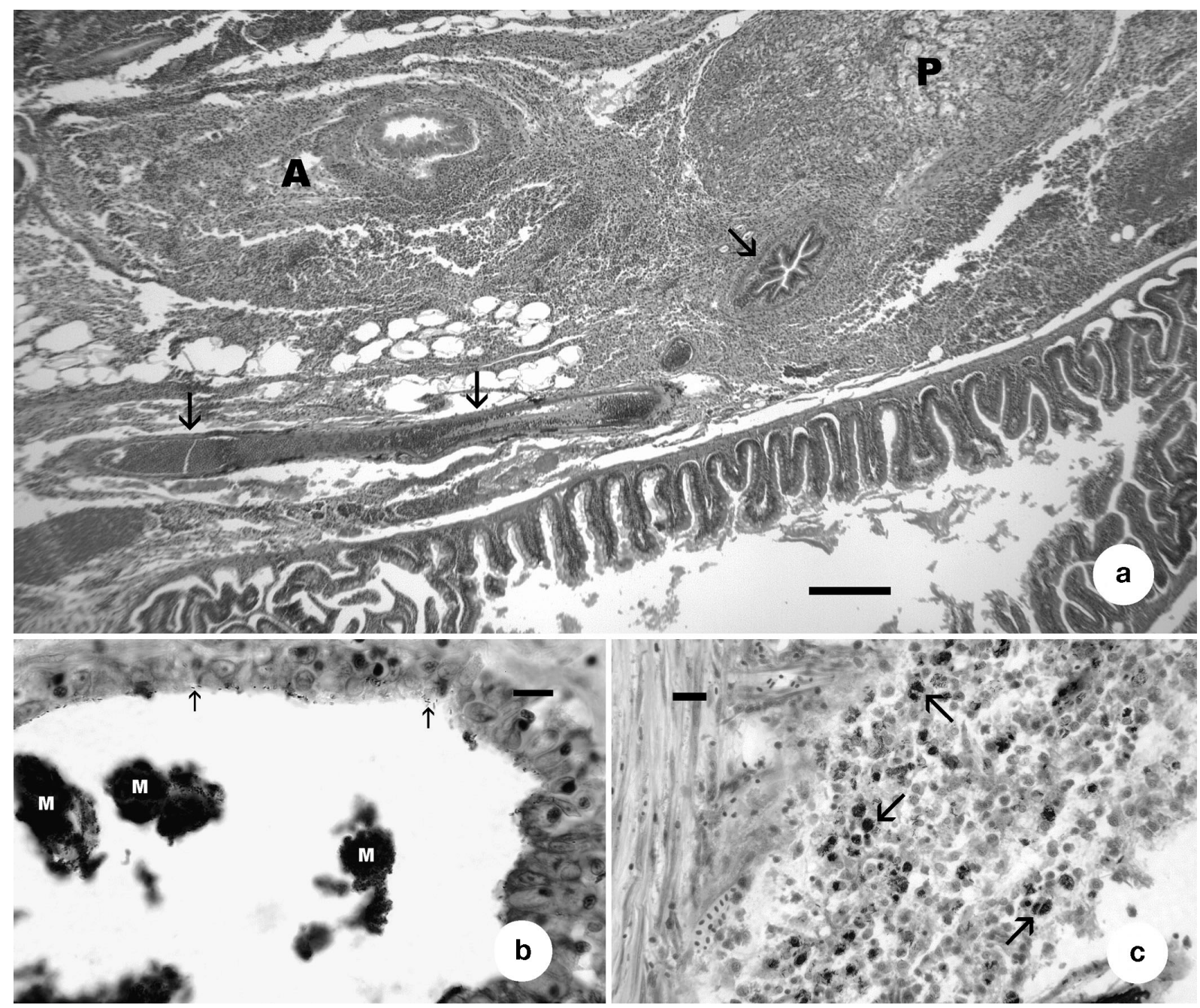

Fig. 2. Mycobacterium chelonae infecting Danio rerio. (a) Sagittal section showing severe, chronic aerocystitis with M. chelonae infection. Anterior $(\mathrm{A})$ and posterior $(\mathrm{P})$ chambers of the swim bladder exhibit massive infiltration of inflammation. Arrows $=$ pneumatic duct connecting to esophagus. Scale bar $=20 \mu \mathrm{m}$. (b) High magnification showing masses of mycobacteria in lumen (M) and in swim bladder wall (arrows). Acid-fast. Scale bar $=10 \mu \mathrm{m}$. (c) Mycobacteria within phagocytes in swim bladder wall (arrows). Scale bar $=10 \mu \mathrm{m}$

\section{Comparison of diagnostic methods}

Histology, culture and PCR all performed comparably well for diagnosis of mycobacteriosis. Histological diagnosis based on H\&E-stained sections was less reliable than acid-fast-stained sections, as diagnosis depended on the observation of granulomas or generalized chronic inflammation, which were present in females with EAIF in the absence of AFB (Table 1). Nevertheless, agreement between H\&E histology and culture was modest $(K=0.6398)$. Diagnosis of mycobacteria by acid-fast-stained sections had substantial agreement $(K=0.7794)$ to culture results. Culture and PCR appear to be more sensitive than observations from tissue sections, as no AFB were observed in 6 of
24 zebrafish positive by PCR or culture. PCR directly from tissues and growth by culture were correlated perfectly.

\section{DISCUSSION}

Our investigation of zebrafish mycobacteriosis identified Mycobacterium chelonae in all positive individuals and the same subtype of $M$. chelonae associated with all but 1 infection. The zebrafish that made up the majority of our culture positive samples (21/24) were the TU genetic line, suggesting these fish are more susceptible to infection. The 3 TU populations were euthanized, and replaced with a new line of TU. This 
was accomplished by following routine procedures for introduction of new lines, in which zebrafish are spawned in a quarantine facility and only progeny from chlorine-disinfected eggs are introduced. The remaining 3 infected zebrafish were from the TL, kita, and WIK lines. Overall, prevalence of mycobacteria in the facility was $10 \%$. However, when TU zebrafish were excluded, prevalence was much lower, at $1.25 \%$. With regards to the higher prevalence of mycobacteriosis in the TU line, the TU line or this particular stock may be genetically more susceptible to mycobacteriosis. Swaim et al. (2006) found that zebrafish lacking the rag1 gene were hypersusceptible to $M$. marinum infection, supporting a genetic basis for susceptibility. In cattle, breed susceptibility to $M$. avium subsp. paratuberculosis has been reported (Koets et al. 2000), but found to have little effect in other studies (Pavlik et al. 2000). As pointed out by Chiodini et al. (1984), breed susceptibility may be difficult to dissect from unique exposure events, leading to an alternate explanation, namely that this particular stock of zebrafish was exposed to high levels of $M$. chelonae, perhaps early in development. Until more is known on the transmission of different mycobacteria in fish, the interpretations of such observations and control of spread is difficult.

Comparing diagnostic methods, there was substantial agreement between culture and histology when sections were stained with H\&E ( $K=0.6398)$. However, in some zebrafish the presence of degenerating eggs and EAIF as described by Kent et al. (2002) led to a misdiagnosis of mycobacteriosis (i.e. granuloma formation is present in both conditions). This serves as a reminder that diagnosis by observation of granuloma is presumptive at best. Conversely, the absence of granulomas would result in an under-appreciation of infection determined by H\&E sections, as fish with mycobacteriosis may exhibit diffuse inflammatory lesions without discrete granulomas (Kent et al. 2004, Swaim et al. 2006). In a similar cross-sectional study of mycobacterial infection in striped bass, Kaattari et al (2005) observed much higher prevalence by PCR or culture than with observation of granuloma in H\&E-stained sections.

When sections were stained acid-fast, the presence or absence of mycobacteria could be clearly distinguished and comparison of culture versus acid-faststained sections had substantial agreement and an increased Kappa coefficient $(K=0.7794)$. Thus, histology with acid-fast staining remains a reliable tool for identification of infected animals; where species identifications are required, culture and PCR-based methods can be employed. Where culture and PCR were negative, only 3 zebrafish were observed to have AFB. These were less severe infections and the discrepancy may be explained by an uneven distribution of the bac- terium throughout the viscera. Unequal distribution may also explain the 6 culture-positive zebrafish that were AFB negative by histology. Alternatively, these positives could have been a result of environmental contamination during necropsy, as the same strains of M. chelonae are present outside of fish. We believe this unlikely for 2 reasons: (1) during necropsy the body surface was wiped with alcohol and great care was taken to avoid puncture of the gut; and (2) other Mycobacterium species isolated from biofilms (Table 2) were never isolated from zebrafish samples. Thus, we are confident of the potential for PCR for the rapid screening of zebrafish mycobacteria in the future.

A major advantage of PCR is its ability to identify Mycobacterium species quickly. A common method for genetic differentiation of mycobacteria is $16 \mathrm{~S}$ small subunit (SSU) rDNA sequencing (Tortoli 2003). We have successfully used SSU rDNA to identify zebrafish mycobacteria from either cultures or directly from DNA extracted from infected host tissues (Kent et al. 2004). However, a limitation of using SSU alone is that a few species have identical SSU rDNA sequences (Tortoli 2003). More variable sequences, such as the internal transcribed spacer (ITS), may be useful for species and strain identification (Roth et al. 1998, 2000) and we have found differences in ITS sequence from 2 M. chelonae isolates from zebrafish that had identical SSU rDNA sequences (Kent et al. 2004). We have also identified a particularly virulent strain of $M$. marinum that possesses a single nucleotide polymorphism at position 5 of the ITS rDNA, which can be used to distinguish it from other isolates (Ostland et al. 2008). For routine identification, however, the heat shock protein 65 gene ( $h$ sp65) appears to be the most reliable (Kim et al. 2005, McNabb et al. 2006), differentiating species that SSU rDNA sequence cannot (Ringuet et al. 1999) and providing greater phylogenetic resolution than the ITS (Whipps et al. 2007b). Here we were able to identify 2 hsp65 sequence types of $M$. chelonae in zebrafish or biofilms. Both hsp65 types were consistent with those of $M$. chelonae we previously isolated from zebrafish, cases 13 and 19 of Kent et al. (2004), subsequently designated HU1 (GenBank \# DQ866784) and HU2 (GenBank \# DQ866785) by Whipps et al. (2007b). Nonetheless, to subdivide strains more thoroughly, additional steps must be taken.

Molecular typing by pulsed-field gel electrophoresis (PFGE) is often used for species of Mycobacterium, including $M$. chelonae (Vanitha et al. 2003). Other methods, such as amplified fragment length polymorphism (AFLP), have also been used to conduct epidemiological studies of mycobacteria (Motiwala et al. 2003) and yield results comparable to PFGE (Willems et al. 2000, D'Agata et al. 2001). In our experience, 
PFGE is reliable but excessively time-consuming (Ostland et al. 2008), while AFLP is inconsistent and difficult to interpret. As a result, we have switched to the more rapid ERIC-PCR. Compared to PFGE, ERIC provides equal, if not greater resolution, and results can be obtained quickly (Sampaio et al. 2006). Furthermore, such analyses can be performed by any lab with a thermocycler and results are interpreted by visual inspection, comparing amplification patterns of various isolates on a single gel. To supplement ERIC-PCR, RAPD analysis is also performed using primers of Zhang et al. (1997). We have found the OPA-2 to be the most discriminatory, as in other studies (Esteban et al. 2007), yielding results equivalent to ERIC-PCR. These are the methods we employ on a routine basis and recommend for delineation of mycobacteria with identical hsp65 genes.

The same strain of Mycobacterium chelonae (H1E2) was identified in all but 2 infected zebrafish, which were infected with $M$. chelonae H1E1 and H1E3. The predominance of a single strain suggests a common source of infection, possibly environmental. In an attempt to identify potential sources or reservoirs of infections in zebrafish, we tested several biofilms for mycobacteria. These included tank surfaces, drains, sump tanks, and Norprene tubing. Mycobacterium chelonae (H1E2) was identified on several tank surfaces, drains, the sump and tubing feeding water to tanks. Fortunately, no mycobacteria were detected in fish food, the main incoming water supply, or from the city water supply to the dishwasher or kitchen faucet. Increased sampling volumes may yet reveal some mycobacteria in these sources. However, for now our results suggest that $M$. chelonae is endemic at the facility, although its pathogenicity and virulence in most lines of zebrafish may be minimal under standard conditions.

Previous experimental transmission studies conducted with Mycobacterium chelonae strains that we have collected and tested to date suggest that $M$. chelonae is less virulent than $M$. marinum and $M$. haemophilium (Watral \& Kent 2007, Whipps et al. 2007a, Ostland et al. 2008). Although we have not yet evaluated H1E2 (the most common isolate from this study) by experimental exposure, the isolate HU1 (type H1E1; also present in the facility) caused little disease following intraperitoneal injections. Harriff et al. (2008) evaluated several strains of $M$. chelonae (including H1E1) and a closely related organism from salmon ( $M$. salmoniphilum) with in vitro macrophage proliferation assays and a microarray. Corresponding to our in vivo study (Watral \& Kent 2007), the H1E1 grew poorly in macrophage cell lines and lacked a newly discovered pathogenicity island (Harriff et al. 2008). The prevalent strain in the present study (H1E2) should be evaluated with these in vivo and in vitro assays.

The manifestation of infection showed a consistent pattern. Histological examinations of infected zebrafish revealed that the occurrence of disease was largely confined to male fish with chronic aerocystitis. Zebrafish have a physostomus swim bladder with a pneumatic duct connecting it to the esophagus (Finney et al. 2006). Harriff et al. (2007) showed that the primary route of infection by mycobacteria in zebrafish is oral; thus, connection with the digestive tract via this duct may provide a route for swim bladder infections. None of the 3 infected females in the current study showed signs of infection in the swim bladder. Thus, the reason for the apparent predisposition of mycobacterial aerocystitis in males here is unknown. In a review of the Zebrafish International Resource Center diagnostic cases, aerocystitis was diagnosed in 35 zebrafish (21 females, 14 males) from 20 groups of fish submitted from research laboratories between 2003 and 2007 . AFB were found in the swim bladder lesions in $51 \%(18 / 35)$ of these fishes. Other cases were associated with Gram-negative bacterial or fungal infections (Kent et al. 2002).

Ultimately, the control of mycobacteriosis in zebrafish colonies first requires a thorough understanding of the virulence diversity, prevalence, distribution and potential modes of transmission for Mycobacterium species found in research facilities. We have seen significant differences in virulence between Mycobacterium species infecting zebrafish, highlighting the importance of species identification (Watral \& Kent 2007, Whipps et al. 2007a). Preventing the introduction of the highly virulent strains and species, particularly M. marinum and $M$. haemophilum, to fish research facilities is paramount, because once established, mycobacteria can be difficult to eliminate (FrancisFloyd \& Yanong 1999). In contrast, M. chelonae may be very common in water and has been isolated from aquaria in the absence of disease (Beran et al. 2006). With these low virulent, ubiquitous strains, dramatic measures such as entire euthanasia of the affected population may not be warranted. However, even with these strains, it would be prudent to minimize the spread of infections. Antibiotic treatment is usually discouraged due to lengthy treatment times and varying susceptibility of Mycobacterium spp. to different antibiotics (Decostere et al. 2004). However, in circumstances where a stock of fish is very valuable, identification of the Mycobacterium sp. by culture or PCR, followed by treatment with the appropriate antibiotic, based on known vulnerability or susceptibility testing, might be advisable upon the recommendation of the facility's laboratory animal veterinarian. Depopulation and disinfection of the facility may be necessary when 
all other measures fail. This unfavorable course of action may be avoidable by minimizing the chances of introduction and spread within a facility. Some measures we recommend are summarized in Kent et al. (2008) and include sentinel programs, quarantine of incoming fish and eggs, regular monitoring, minimizing circulating mycobacteria through regular cleaning and maintenance, and upkeep of the UV sterilization system.

In conclusion, this survey of background levels of Mycobacterium species in a zebrafish facility revealed that $M$. chelonae was the only species isolated from zebrafish. The same strains were identified in surface biofilms, implicating a potential environmental reservoir of infection. Overall, levels of infection in the facility were very low, with the exception of TU zebrafish, which represented all but 3 of our positive fish. Thus, we suspect that TU zebrafish are more susceptible to infection. Consistent with results from our earlier virulence experiments (Watral \& Kent 2007), virulence of M. chelonae appeared to be low, i.e. few fish showed signs of overt mycobacteriosis. This study demonstrates that $M$. chelonae infections can persist in the background, even at very well-managed facilities. These results emphasize the importance of good husbandry and fish health monitoring in zebrafish research facilities to minimize the risk of a disease outbreak, with the ultimate goal of greatly reducing or eliminating these infections from laboratory-reared populations.

Acknowledgements. This work was supported by National Institute of Health (NIH) Grant NCRR 5R24RR017386-02 and P40 RR12546. C.M.W. is grateful for the services of Nevada Genomics Center, which is supported in part by a grant from the Nevada IDeA Network of Biomedical Research Excellence (2 P20 RR016463).

\section{LITERATURE CITED}

Astrofsky KM, Schrenzel MD, Bullis RA, Smolowitz RM, Fox JG (2000) Diagnosis and management of atypical Mycobacterium spp. infections in established laboratory zebrafish (Brachydanio rerio) facilities. Comp Med 50:666-672

Baker D (2003) Natural pathogens of laboratory animals: their effects on research. ASM Press, Herdon, VA

> Beran V, Matlova L, Dvorska L, Svastova P, Pavlik I (2006) Distribution of mycobacteria in clinically healthy ornamental fish and their aquarium environment. J Fish Dis 29 383-393

> Chiodini RJ, van Kruiningen HJ, Merkal RS (1984) Ruminant paratuberculosis (Johne's disease): the current status and future prospects. Cornell Vet 74:218-262

> Cohen J (1960) A coefficient of agreement for nominal scales. Educ Psychol Meas 20:37-46

> D'Agata EM, Gerrits MM, Tang YW, Samore M, Kusters JG (2001) Comparison of pulsed-field gel electrophoresis and amplified fragment-length polymorphism for epidemiological investigations of common nosocomial pathogens. Infect Control Hosp Epidemiol 22:550-554
Decostere A, Hermans K, Haesebrouck F (2004) Piscine mycobacteriosis: a literature review covering the agent and the disease it causes in fish and humans. Vet Microbiol 99:159-166

Esteban J, Fernández Roblas R, García Cía JI, Zamora N, Ortiz A (2007) Clinical significance and epidemiology of non-pigmented rapidly growing mycobacteria in a university hospital. J Infect 54:135-145

Finney JL, Robertson GN, McGee CAS, Smith FM, Croll RPJ (2006) Structure and autonomic innervation of the swim bladder in the zebrafish (Danio rerio). J Comp Neurol 495: $587-606$

Francis-Floyd R, Yanong RPE (1999) Mycobacteriosis in fish. Fact Sheet VM-96. Institute of Food and Agricultural Sciences, University of Florida, Gainsville, FL

Harriff MJ, Bermudez LE, Kent ML (2007) Experimental exposure of zebrafish, Danio rerio (Hamilton), to Mycobacterium marinum and Mycobacterium peregrinum reveals the gastrointestinal tract as the primary route of infection: a potential model for environmental mycobacterial infection. J Fish Dis 30:587-600

Harriff MJ, Wu M, Kent ML, Bermudez LE (2008) Species of environmental mycobacteria differ in their abilities to grow in human, mouse, and carp macrophages and with regard to the presence of mycobacterial virulence genes, as observed by DNA microarray hybridization. Appl Environ Microbiol 74:275-285

> Kaattari IM, Rhodes MW, Kator H, Kaattari SL (2005) Comparative analysis of mycobacterial infections in wild striped bass Morone saxatilis from Chesapeake Bay. Dis Aquat Org 67:125-132

Kent PT, Kubica GP (1985). Public health mycobacteriology: a guide for the Level III laboratory. US Dept Public Health \& Human Services, Center for Disease Control, Atlanta, GA

Kent ML, Spitsbergen JM, Matthews JL, Fournie JW, Westerfield M (2002) Diseases of zebrafish in research facilities. Available at: http://zebrafish.org/zirc/health/ diseaseManual.php (accessed March 26, 2008)

Kent ML, Whipps CM, Matthews JL, Florio D and others (2004) Mycobacteriosis in zebrafish (Danio rerio) research facilities. Comp Biochem Phys C 138:383-390

Kent ML, Feist SW, Harper C, Hoogstraten-Miller S and others (2008) Recommendations for control of pathogens and infectious diseases in fish research facilities. Comp Biochem Phys C (in press)

Kim H, Kim SH, Shim TS, Kim MN and others (2005) Differentiation of Mycobacterium species by analysis of the heatshock protein 65 gene (hsp65). Int J Syst Evol Microbiol 55:1649-1656

Koets AP, Adugna G, Janss LL, van Weering HJ and others (2000) Genetic variation of susceptibility to Mycobacterium avium subsp. paratuberculosis infection in dairy cattle. J Dairy Sci 83:2702-2708

Landis JR, Koch GG (1977) The measurement of observer agreement for categorical data. Biometrics 33:159-174

McNabb A, Adie K, Rodrigues M, Black WA, Isaac-Renton J (2006) Direct identification of mycobacteria in primary liquid detection media by partial sequencing of the 65kilodalton heat shock protein gene. J Clin Microbiol 44: 60-66

Motiwala AS, Strother M, Amonsin A, Byrum B and others (2003) Molecular epidemiology of Mycobacterium avium subsp. paratuberculosis: evidence for limited strain diversity, strain sharing, and identification of unique targets for diagnosis. J Clin Microbiol 41:2015-2026

> Ostland VE, Watral VG, Whipps CM, Austin F, St. Hilaire S, Westerman ME, Kent ML (2008) Biochemical, molecular, 
and virulence studies of select Mycobacterium marinum strains in hybrid striped bass Morone chrysops $\times$ M. saxatilis and zebrafish Danio rerio. Dis Aquat Org 79:107-118

Pavlik I, Matlova L, Bartl J, Svastova P, Dvorska L, Whitlock R (2000) Parallel faecal and organ Mycobacterium avium subsp. paratuberculosis culture of different productivity types of cattle. Vet Microbiol 77:309-324

Poort MJ, Whipps CM, Watral VG, Font WF, Kent ML (2006) Molecular characterization of a Mycobacterium species in non-native poeciliids in Hawaii using DNA sequences. J Fish Dis 29:181-185

Ringuet H, Akoua-Koffi C, Honore S, Varnerot A and others (1999) hsp65 sequencing for identification of rapidly growing mycobacteria. J Clin Microbiol 37:852-857

Roth A, Fischer M, Hamid ME, Michalke S, Ludwig W, Mauch H (1998) Differentiation of phylogenetically related slowly growing mycobacteria based on 16S-23S rDNA gene internal transcribed spacer sequences. J Clin Microbiol 36:139-147

Roth A, Reischl U, Streubel A, Naumann L and others (2000) Novel diagnostic algorithm for identification of mycobacteria using genus-specific amplification of the 16S-23S rRNA gene spacer and restriction endonucleases. J Clin Microbiol 38:1094-1104

Sampaio JL, Viana-Niero C, de Freitas D, Hofling-Lima AL, Leao SC (2006) Enterobacterial repetitive intergenic consensus PCR is a useful tool for typing Mycobacterium chelonae and Mycobacterium abscessus isolates. Diagn Microbiol Infect Dis 55:107-118

Swaim LE, Connolly LE, Volkman HE, Humbert O, Born DE, Ramakrishnan L (2006) Mycobacterium marinum infection

Editorial responsibility: Catherine Collins, Aberdeen, UK of adult zebrafish causes caseating granulomatous tuberculosis and is moderated by adaptive immunity. Infect Immun 74:6108-6117

Tortoli E (2003) Impact of genotypic studies on mycobacterial taxonomy: the new mycobacteria of the 1990s. Clin Microbiol Rev 16:319-354

Vanitha JD, Venkatasubramani R, Dharmalingam K, Paramasivan CN (2003) Large-restriction-fragment polymorphism analysis of Mycobacterium chelonae and Mycobacterium terrae isolates. Appl Environ Microbiol 69:4337-4341

Watral V, Kent ML (2007) Pathogenesis of Mycobacterium spp. in zebrafish (Danio rerio) from research facilities. Comp Biochem Phys C 145:55-60

Westerfield M (2000) The zebrafish book: a guide for the laboratory use of zebrafish (Danio rerio), 4th edn. University of Oregon Press, Eugene, OR

Whipps CM, Dougan ST, Kent ML (2007a) Mycobacterium haemophilum infections of zebrafish (Danio rerio) in research facilities. FEMS Microbiol Lett 270:21-26

Whipps CM, Butler WR, Pourahmad F, Watral VG, Kent ML (2007b) Molecular systematics support the revival of Mycobacterium salmoniphilum (ex Ross 1960) sp. nov., nom. rev., a species closely related to Mycobacterium chelonae. Int J Syst Evol Microbiol 57:2525-2531

Willems RJ, Top J, van Den Braak N, van Belkum A and others (2000) Host specificity of vancomycin-resistant Enterococcus faecium. J Infect Dis 182:816-823

Zhang Y, Rajagopalan M, Brown BA, Wallace RJ Jr (1997) Randomly amplified polymorphic DNA PCR for comparison of Mycobacterium abscessus strains from nosocomial outbreaks. J Clin Microbiol 35:3132-3139

Submitted: March 31, 2008; Accepted: July 14, 2008 Proofs received from author(s): September 17, 2008 